\title{
Supervivencia según vía de abordaje quirúrgico en pacientes con adenocarcinoma de endometrio tratadas en Navarra en el periodo 2001-2009
}

\section{Survival by surgical approach in patients with endometrial adenocarcinoma treated in Navarra in the 2001-2009}

\author{
M. Ibarrola Vidaurre ${ }^{1}$, J. C. Muruzábal Torquemada ${ }^{2}$, S. Aguirre Gorospe ${ }^{2}$, \\ V. Segura Ortega ${ }^{2}$, O. Tarrio Fernández ${ }^{2}$, S. Lapeña Calavia ${ }^{2}$
}

\section{RESUMEN}

Fundamento. El adenocarcinoma de endometrio es la neoplasia ginecológica más frecuente tras el cáncer de mama y representa el $6 \%$ de todos los cánceres de la mujer. El tratamiento fundamental de dicha enfermedad es la cirugía. La mayoría de los casos se diagnostican en estadios iniciales y la cirugía será curativa; en otras ocasiones es necesario añadir radioterapia o quimioterapia. El tratamiento clásico del adenocarcinoma de endometrio es la histerectomía con doble anexectomía por vía laparotómica, añadiendo según las características del caso la linfadenectomía pélvica, paraaórtica y omentectomía. Durante los últimos 10-15 años se ha introducido la laparoscopia en el tratamiento quirúrgico del adenocarcinoma de endometrio.

El objetivo principal de este trabajo es analizar los casos de adenocarcinoma de endometrio intervenidos quirúrgicamente en el antiguo hospital Virgen de Camino (hoy Complejo Hospitalario de Navarra) durante el periodo 2001-2009.

Material y métodos. Se ha recogido una cohorte histórica de 444 pacientes con diagnóstico de adenocarcinoma de endometrio durante el periodo 2001-2009, que recibieron tratamiento quirúrgico, así como su seguimiento durante 4 años.

Conclusiones. Los resultados confirman que la vía laparoscópica es una alternativa segura a la laparotomía clásica ya que no afecta a la supervivencia ni al tiempo libre de enfermedad tanto en el adenocarcinoma endometrioide como en el no endometrioide.

Palabras clave. Carcinoma de endometrio. Laparoscopia. Laparotomía. Supervivencia. Tiempo libre de enfermedad.

\begin{abstract}
Background. Endometrial adenocarcinoma is the most frequent gynaecological neoplasia after breast cancer and represents $6 \%$ of cancers in women. The treatment for this disease is surgery. The majority of cases are diagnosed in their initial stages and surgery is curative; on other occasions it is necessary to add radiotherapy and chemotherapy. The classical treatment for endometrial adenocarcinoma is hysterectomy with double adnexectomy by laparotomy, with the addition of pelvic and para-aortic lymphadenectomy and omentectomy according to the characteristics of the case. During the last 10-15 years laparoscopy has been introduced in the surgical treatment of endometrial adenocarcinoma.

The main aim of this study is to analyze the cases of endometrial adenocarcinoma treated surgically in the former Virgen de Camino Hospital (nowadays the Hospital Complex of Navarra) during 2001-2009.
\end{abstract}

Methods. Historical cohort of 444 patients with endometrial adenocarcinoma during 2001-2009 who received surgical treatment, followed four years.

Conclusions. The results confirm that laparoscopy is a safe alternative to classical laparotomy as it does not affect either survival or time free of disease, in both endometrioid adenocarcinoma and non-endometrioid adenocarcinoma.

Keywords. Endometrial carcinoma. Laparoscopy. Laparotomy. Survival. Disease-free survival.
1. Servicio Obstetricia y Ginecología. Hospital García Orcoyen. Estella.

2. Servicio de Obstetricia y Ginecología. Complejo Hospitalario de Navarra. Pamplona.

Recepción: 30 de julio de 2014

Aceptación provisional: 10 de septiembre de 2014

Aceptación definitiva: 22 de octubre de 2014
Correspondencia:

María Ibarrola Vidaurre

Servicio de Obstetricia y Ginecología

Hospital García Orcoyen

Santa Soria, 22

31200 Estella. Navarra

E-mail: maria.ibarrola.vidaurre@cfnavarra.es 


\section{INTRODUCCIÓN}

El adenocarcinoma de endometrio es la neoplasia ginecológica más frecuente tras el cáncer de mama y representa el $6 \%$ de todos los cánceres de la mujer. Es una enfermedad potencialmente curable aunque dependerá de la extirpe celular, el estadio de la enfermedad y otros factores pronósticos $^{1}$.

Pueden diferenciarse dos grandes grupos que marcarán el pronóstico de la enfermedad. En primer lugar el adenocarcinoma de tipo endometrioide (75-80\%), con buen pronóstico, la mayor parte diagnosticados en estadios iniciales, y el adenocarcinoma de tipo no endometriode (20-25\%), con un comportamiento más agresivo similar al cáncer de ovario ${ }^{1}$.

El tratamiento más frecuente de esta enfermedad es la cirugía ${ }^{1}$. La mayoría de los casos se encuentran en estadios iniciales y la cirugía será curativa. En ocasiones, es necesario añadir radioterapia o quimioterapia. El tratamiento clásico del adenocarcinoma de endometrio ha sido la histerectomía con doble anexectomía por vía laparotómica añadiendo, según las características del caso, la linfadenectomía pélvica, paraaórtica y omentectomía.

Durante los últimos 10-15 años se ha introducido la laparoscopia en el tratamiento quirúrgico del adenocarcinoma de endometrio. Este cambio en el abordaje quirúrgico obliga a demostrar que la laparoscopia es una alternativa segura $a^{2-5}$ y con similares o mejores resultados ${ }^{2-4,6,7}$ para las pacientes sin verse afectada la seguridad oncológica ${ }^{8}$.

El objetivo principal en este estudio es analizar los casos de adenocarcinoma de endometrio intervenidos quirúrgicamente en el antiguo hospital Virgen de Camino (hoy Complejo Hospitalario de Navarra) durante el periodo 2001-2009. Dicho periodo abarca el tiempo de transición del abordaje laparotómico a laparoscópico y de centralización de los tumores ginecológicos de Navarra. Es preciso asegurar que el cambio en la vía de abordaje de todos los cánceres de endometrio susceptibles a tratamiento quirúrgico, tanto endome- trioides como no endometrioides y en todos sus estadios, no ha influido en la seguridad oncológica, es decir no habiéndose afectado la supervivencia global ni el tiempo libre de enfermedad según la vía de abordaje.

\section{MATERIAL Y MÉTODOS}

Se realiza el estudio en una cohorte histórica de 444 pacientes con diagnóstico de adenocarcinoma de endometrio durante el periodo 2001-2009, que recibieron tratamiento quirúrgico en el Complejo Hospitalario de Navarra, así como su seguimiento durante 4 años.

Todas las pacientes diagnosticadas de adenocarcinoma de endometrio son candidatas a tratamiento quirúrgico en el Complejo Hospitalario de Navarra salvo criterios de exclusión, definidos por la contraindicación anestésica, mal estado general, edad o enfermedad en estadio IV. Todas ellas recibieron tratamiento mediante una de las dos vías (193 por vía laparotómica y 251 por vía laparoscópica) durante el periodo 2001-2009.

Se recogieron las siguientes variables:

- Edad, índice de masa corporal (IMC), tipo de tumor en biopsia, grado de tumor en biopsia, estadiaje prequirúrgico, pruebas de imagen, tipo de tumor en pieza quirúrgica, grado de tumor en pieza quirúrgica, afectación de ganglios pélvicos, afectación ganglios paraórticos, estadio postquirúrgico, tratamiento adyuvante.

- Fecha de la cirugía, tipo de cirugía, vía de abordaje, días de ingreso.

- Supervivencia (estado de las pacientes: viva/muerta y tiempo hasta evento en los 4 años de seguimiento: ya sea tiempo de seguimiento en los vivos y tiempo hasta fallecimiento en los fallecidos, así como la causa de muerte (cáncer endometrio/ otras).

- Recidiva (si ha habido o no recidiva, y tiempo hasta el evento: tiempo de seguimiento y tiempo hasta la recidiva en los 4 años de seguimiento). 
Las pacientes con el diagnóstico de adenocarcinoma de endometrio mediante biopsia endometrial son remitidas a la sección de Ginecología Oncológica del Complejo Hospitalario de Navarra. Posteriormente se someten a un estudio preoperatorio realizando a través del mismo una estadificación inicial, lo que condiciona el tratamiento quirúrgico. Éste se basa en el tipo histológico, grado de la biopsia inicial y el estudio radiológico (ecografía, RMN y/o TAC según las características de cada caso) para la valoración de la infiltración miometrial ${ }^{1}$.

Tras la estadificación inicial se oferta el tratamiento oportuno basado en la cirugía salvo criterios de exclusión citados previamente. Desde el año 2001 se introduce el abordaje laparoscópico de dicha patología en nuestro centro quedando relegada la laparotomía para casos con tumoraciones pélvicas de gran tamaño, antecedente de cirugías previas, vía vaginal insuficiente para extracción de la pieza quirúrgica, así como la obesidad y la necesidad de linfadenectomía paraórtica; estas dos últimas indicaciones con el paso de los años no fueron motivo de dicho cambio de vía. Al finalizar el tratamiento se realiza seguimiento continuado en consulta quedando reflejados los datos en la historia clínica informatizada, a través de la cual se recabó la información tanto del ingreso como de la evolución posterior para realizar el análisis oportuno.

\section{Análisis de datos}

Las variables cuantitativas se describen mediante medias y desviaciones típicas o medianas y rangos intercuartílicos, según corresponda, y las variables cualitativas mediante frecuencias y porcentajes. Para valorar la asociación entre las variables categóricas (incluido el tipo de abordaje) se utilizó el test $\mathrm{X}^{2}$ de Pearson, el test $\mathrm{X}^{2}$ de tendencias en las proporciones o el test de Fisher, mientras que para comparar la relación entre las variables cuantitativas se usó el test t-Student o el test de Mann-Whitney. Los mismos test se utilizaron para valorar si existían diferen- cias en las características de las pacientes según fuera el tipo de vía laparotómica o laparoscópica. Las diferencias en la evolución del tiempo libre de enfermedad y de supervivencia se compararon mediante el test del log-rank.

En el análisis multivariante la entrada de variables se realizó siguiendo criterios de significación estadística (por cambios en la verosimilud del modelo) y de control de la confusión (valorando los cambios en los coeficientes de regresión con la entrada/salida de cada variable). La medida de asociación fue la OddsRatio (OR) acompañada de su correspondiente intervalo de confianza del $95 \%$.

El tiempo libre de progresión y de supervivencia se valoró con un modelo de regresión de Cox, con los mismos criterios de selección (significación estadística y control de la confusión) comprobando la asunción de proporcionalidad de riesgos. La medida de asociación fue el Hazard Ratio (HR) acompañado del intervalo de confianza del $95 \%$.

Se seleccionó en todos los contrastes un nivel de riesgo alfa del 5\% $(\mathrm{p}<0,05)$. Los análisis se realizarán utilizando el paquete estadístico SPSS, versión 21.

Este estudio fue aprobado por el Comité Ético del Complejo Hospitalario de Navarra.

\section{RESULTADOS}

Se reclutaron 444 pacientes con criterios de tratamiento quirúrgico, 405 con adenocarcinoma endometroide $(91,2 \%)$ y 39 (8,8\%) no endometroide.

La laparoscopia fue la vía de abordaje quirúrgico más utilizada (251 pacientes: $56,5 \%$ ), frente a la laparotomía (193 pacientes: $43,5 \%)$.

En la tabla 1 se describen las características de cada grupo según la vía de abordaje quirúrgico. La edad media fue de 63,8 años (DE 10,8; rango de 32 a 88 años). La edad fue significativamente mayor entre las intervenidas por laparotomía respecto a las abordadas por laparoscopia; 66,3 (DE 10,5 rango de 32 a 88 años) vs 61,9 (DE 10,6 rango de 35 a 86 años); $\mathrm{p}<0,001$. 
Tabla 1. Análisis descriptivo de grupos comparativos de estudio

\begin{tabular}{|c|c|c|c|}
\hline & Global & Laparoscopia & Laparotomía \\
\hline \multirow[t]{3}{*}{ Edad } & 63,8 años (DE & 61,9 años (DE & 66,3 años (DE \\
\hline & 10,8 ; rango de & 10,6 rango de & 10,5 rango de \\
\hline & 32 a 88 años) & 35 a 86 años) & 32 a 88 años) \\
\hline \multicolumn{4}{|l|}{ Tipo de cáncer } \\
\hline Endometrioide & $405(91,2 \%)$ & $241(96,0 \%)$ & $164(85,0 \%)$ \\
\hline No endometrioide & $39(8,8 \%)$ & $10(4,0 \%)$ & $29(15,0 \%)$ \\
\hline \multicolumn{4}{|l|}{ Grado Postoperatorio } \\
\hline Grado I & $194(43,7 \%)$ & $132(52,6 \%)$ & $62(32.1 \%)$ \\
\hline Grado II & $162(36,5 \%)$ & $90(35,9 \%)$ & $72(37,3 \%)$ \\
\hline Grado III & $88(19,8 \%)$ & $29(11,6 \%)$ & $59(30,6 \%)$ \\
\hline \multicolumn{4}{|l|}{ Estadio Postoperatorio } \\
\hline Ia & $320(72,1 \%)$ & $200(79,7 \%)$ & $120(62,2 \%)$ \\
\hline Ib & $58(13,1 \%)$ & $28(11,2 \%)$ & $30(15,5 \%)$ \\
\hline II & $20(4,5 \%)$ & $6(2,4 \%)$ & $14(7,3 \%)$ \\
\hline IIIa & $7(1,6 \%)$ & $3(1,2 \%)$ & $4(2,1 \%)$ \\
\hline IIIb & $14(3,2 \%)$ & $4(1,6 \%)$ & $10(5,2 \%)$ \\
\hline IIIc1 & $11(2,5 \%)$ & $6(2,4 \%)$ & $5(2,6 \%)$ \\
\hline IIIc2 & $9(2,0 \%)$ & $3(1,2 \%)$ & $6(3,1 \%)$ \\
\hline IVa & $2(0,5 \%)$ & 0 & $2(1,0 \%)$ \\
\hline $\mathrm{IVb}$ & $3(0,7 \%)$ & $1(0,4 \%)$ & $2(1,0 \%)$ \\
\hline \multicolumn{4}{|l|}{ Afectación ganglionar } \\
\hline Positivos & $25(5,6 \%)$ & $10(4,0 \%)$ & $15(7,8 \%)$ \\
\hline Negativos & $308(69,4 \%)$ & $211(84,1 \%)$ & $97(50,3 \%)$ \\
\hline Desconocidos & $111(25,0 \%)$ & $30(12,0 \%)$ & $81(42,0 \%)$ \\
\hline \multicolumn{4}{|l|}{ Intervención realizada } \\
\hline Histerectomía con doble anexectomía & $122(27,5 \%)$ & $32(12,7 \%)$ & $90(46,6 \%)$ \\
\hline $\begin{array}{l}\text { Histerectomía con doble anexectomía y linfadenectomía } \\
\text { pélvica }\end{array}$ & $245(55,2 \%)$ & $184(73,3 \%)$ & $61(31,6 \%)$ \\
\hline $\begin{array}{l}\text { Histerectomía con doble anexectomía y linfadenectomía } \\
\text { pélvica y paraaórtica }\end{array}$ & $54(12,2 \%)$ & $33(13,1 \%)$ & $21(10,9 \%)$ \\
\hline $\begin{array}{l}\text { Histerectomía con doble anexectomía, linfadenectomía } \\
\text { pélvica y paraaórtica y omentectomía }\end{array}$ & $23(5,2 \%)$ & $2(0,8 \%)$ & $21(10,9 \%)$ \\
\hline Estancia hospitalaria & $8,9 \pm 5,0$ días & $6,8 \pm 3,8$ días & $11,6 \pm 5,1$ días \\
\hline \multicolumn{4}{|l|}{ Tratamiento posterior } \\
\hline Ninguno & $208(46,8 \%)$ & $142(56,6 \%)$ & $66(34,2 \%)$ \\
\hline Radioterapia pélvica & $16(3,6 \%)$ & $5(2 \%)$ & $11(5,7 \%)$ \\
\hline Quimioterapia & $3(0,7 \%)$ & $1(0,4 \%)$ & $2(1,0 \%)$ \\
\hline Radioterapia + Quimioterapia & $6(1,4 \%)$ & $1(0,4 \%)$ & $5(2,6 \%)$ \\
\hline Radioterapia + Braquiterapia & $100(22,5 \%)$ & $40(15,9 \%)$ & $60(31,1 \%)$ \\
\hline Braquiterapia & $84(18,9 \%)$ & $51(20,3 \%)$ & $33(17,1 \%)$ \\
\hline Radioterapia + Braquiterapia + Quimioterapia & $26(5,9 \%)$ & $11(4,4 \%)$ & $15(7,8 \%)$ \\
\hline Hormonoterapia & $1(0,2 \%)$ & 0 & $1(0,5 \%)$ \\
\hline
\end{tabular}

En la estadificación inicial, previo a la cirugía, en el $86,5 \%$ de los casos se trataba de un adenocarcinoma endometrioide frente a un $7,7 \%$ de no endometrioide. En un $4,3 \%$ no se disponía de biopsia previa y fue un hallazgo casual en la cirugía o se te- nía una alta sospecha diagnóstica. En 1,6\% de los casos el diagnóstico era hiperplasia compleja con atipias en el límite con adenocarcinoma.

Debido a la estadificación inicial así como a posibles dificultades en el acto 
quirúrgico se realizaron las diferentes variantes quirúrgicas. En el $55,2 \%$ de los casos se realizó la cirugía clásica (histerectomía con doble anexectomía) junto a la linfadenectomía pélvica con fines terapéuticos y diagnósticos. Cabe destacar que dicha modalidad quirúrgica se realizó en el $73,3 \%$ de las pacientes intervenidas por laparoscopia, mientras que en las que se intervino por vía laparotómica solo alcanzó el $31,6 \%$. En la vía laparotómica la cirugía predominante fue la clásica (histerectomía con doble anexectomía) en el $46,6 \%$ de los casos.

Tras la cirugía correspondiente se obtuvo la estadificación definitiva lo que permitió la valoración del tratamiento complementario. Como se describe en la literatura actual, el $91,2 \%$ se trata de adenocarcinomas de tipo endometrioide y en estadios iniciales (85,2\%). La afectación ganglionar está presente en el 5,1\% de los casos, siendo más frecuente la afectación pélvica $(3,8 \%)$ que paraaórtica $(2,7 \%)$.

Algo más de la mitad de las pacientes $(53,2 \%)$ necesitaron tratamiento complementario, siendo la braquiterapia junto a radioterapia externa el más usado, seguido por la braquiterapia aislada. El uso de quimioterapia fue escaso, en torno al $6 \%$ de los casos, quedando relegada para los tumores no endometrioides.

El 14,4\% de las pacientes presentaron una recidiva tumoral a distancia siendo la más frecuente la afectación pulmonar $(21,2 \%)$, seguida de la hepática $(17,3 \%)$ y ósea $(9,6 \%)$.

En la figura 1 puede observarse la tasa acumulada de casos de progresión de la enfermedad en relación al tipo de abordaje quirúrgico. Al diferenciarlos según el tipo de tumor la significación estadística se pierde en los no endometrioides (log rank: $\mathrm{p}=0,66$ ), no así en los endometrioides (log rank: $\mathrm{p}=0,017$ ). Inicialmente se realiza un análisis bivariante mediante el test de $\mathrm{Ka}$ plan Meyer cuyos resultados pueden verse en la tabla 2.1. Sin ajustarlo por los factores de confusión parecen tener mejor pronóstico las pacientes intervenidas por laparoscopia. Tras realizar el análisis multivariante y ajustarlo por los factores de confusión como son la edad, el tipo de tumor, el grado y el estadio, concluimos que el tiempo libre de enfermedad no depende del tipo de abordaje quirúrgico realizado. Una mayor edad, el grado y estadio de la enfermedad son factores de riesgo que incrementan el riesgo de recaída, acortando el tiempo libre de enfermedad (Tabla 2.2).

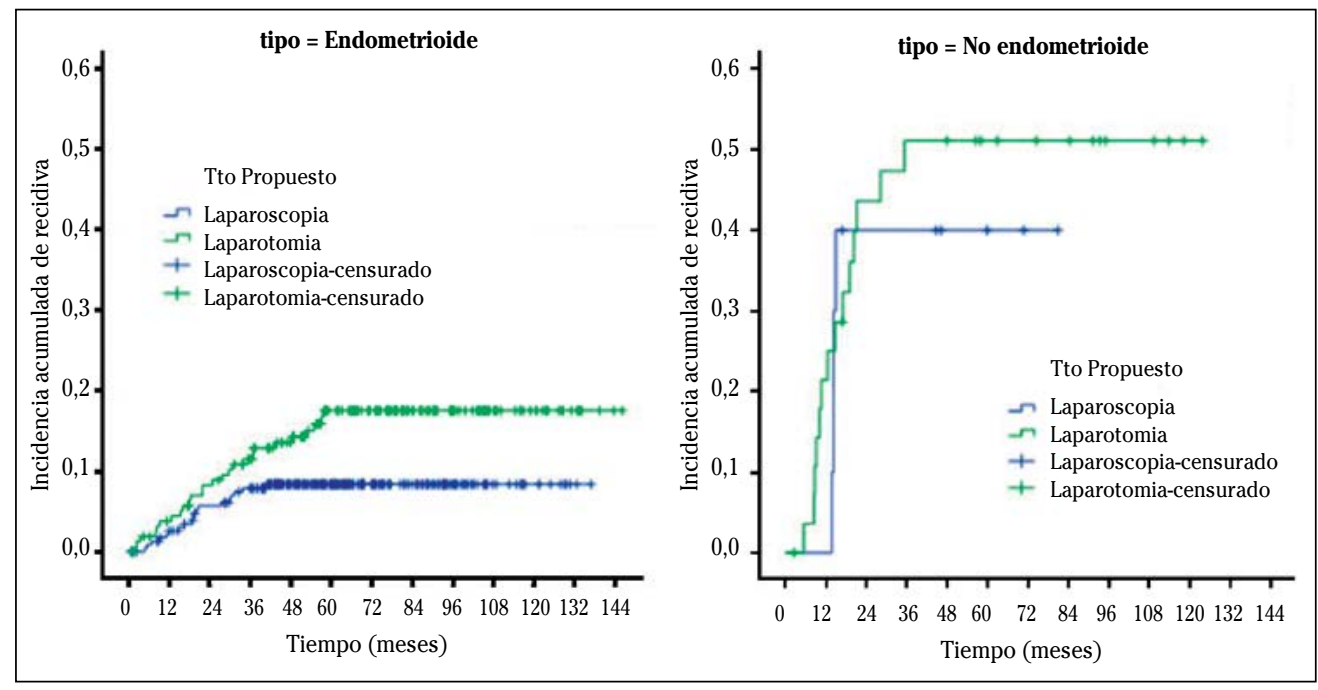

Figura 1. Tiempo libre de progresión. 
Tabla 2. Tiempo libre de enfermedad

2.1 Análisis bivariante (Kaplan Meyer)

\begin{tabular}{|c|c|c|}
\hline & $\begin{array}{c}\text { Meses de TLE } \\
\text { Media (IC95\%)* }\end{array}$ & $\mathbf{p}$ \\
\hline Abordaje & & 0,001 \\
\hline Laparotomía & $119(111$ a 126$)$ & \\
\hline Laparoscopia & $125,5$ (121 a 130$)$ & \\
\hline Edad & & $<0,001$ \\
\hline$<65$ años & 130 (126 a 135) & \\
\hline$\geq 65$ años & $117(110$ a 124$)$ & \\
\hline Tipo de cáncer & & $<0,001$ \\
\hline Endometroide & 131 (127 a 135) & \\
\hline No endometroide & 71 (53 a 89) & \\
\hline Grado & & $<0,001$ \\
\hline G1 & 135 (130 a 139) & \\
\hline $\mathrm{G} 2$ & 115 (105 a 124) & \\
\hline G3 & 99 (87 a 112) & \\
\hline Estadio FIGO & & $<0,001$ \\
\hline I & 133 (129 a 137) & \\
\hline II & $90(79$ a 104$)$ & \\
\hline III & $83(64$ a 101) & \\
\hline IV & $38(25$ a 52$)$ & \\
\hline
\end{tabular}

* Media de supervivencia al no poderse estimar la mediana en todos estratos

2.2 Análisis multivariante (Regresión de Cox)

\begin{tabular}{lcc}
\hline & HR (IC95\%) & p \\
\hline $\begin{array}{l}\text { Abordaje } \\
\text { Laparotomía (ref) } \\
\text { Laparoscopia }\end{array}$ & $0,86(0,50-1,49)$ & 0,60 \\
\hline Edad (incr. 1 año) & $1,047(1,018-1,077)$ & $<0,001$ \\
\hline Tipo de cáncer & & 0,51 \\
$\quad$ Endometroide (ref) & 1 & \\
$\quad$ No endometroide & $1,27(0,63-2,56)$ & \\
\hline Grado & & $<0,001$ \\
$\quad$ I (ref) & 1 & \\
II & $4,52(1,70-12,0)$ & \\
III & $9,62(3,42-27,1)$ & \\
\hline Estadio FIGO & & $<0,001$ \\
$\quad$ I (ref) & 1 & \\
II & & \\
III & $0,73(0,25-2,11)$ & \\
IV & $2,06(1,11-3,83)$ & \\
\hline
\end{tabular}

HR: hazard ratio 
En la figura 2 se muestra el análisis de la supervivencia sin ajustarlo por los factores de confusión. Al igual que al analizar el tiempo libre de enfermedad y desglosarlo según el tipo de tumor se pierde la significación estadística en los tumores no endometrioides (long rank: $\mathrm{p}=0,74$ ), no así en los endometrioides (log rank: $\mathrm{p}=0,039)$.
En la tabla 3.1 se muestra en análisis bivariante mediante el test de Kaplan Meyer. Y al realizar el análisis multivariante y ajustarlo por los mismos factores de confusión (edad, tipo de tumor, grado y estadio de la enfermedad) comprobamos que el tiempo de supervivencia no depende del tipo de abordaje quirúrgico realizado (Tabla 3.2).

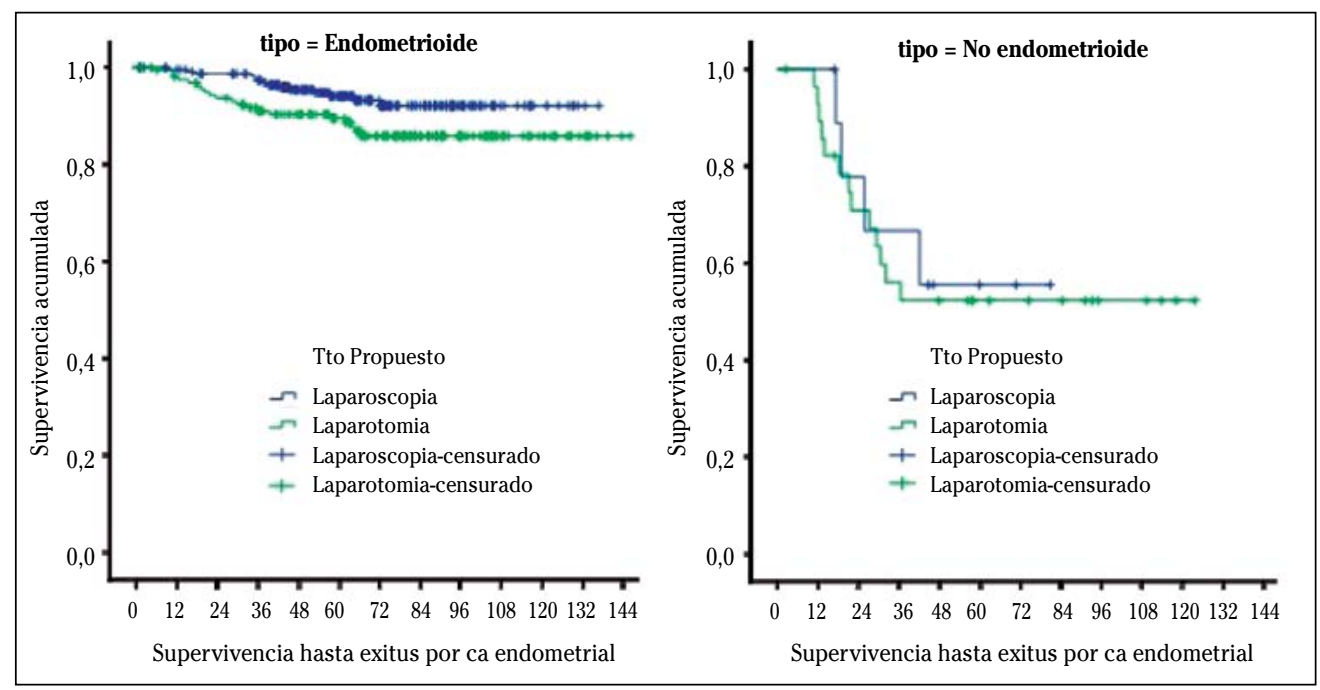

Figura 2. Tiempo de supervivencia global.

Tabla 3. Supervivencia

3.1 Análisis bivariante (Kaplan Meyer)

\begin{tabular}{lcc}
\hline & $\begin{array}{c}\text { Meses de supervivencia } \\
\text { Media (IC95\%)* }\end{array}$ & p \\
\hline $\begin{array}{l}\text { Abordaje } \\
\text { Laparotomía }\end{array}$ & $111(103$ a 119$)$ & 0,009 \\
$\quad$ Laparoscopia & $117(111$ a 123$)$ & \\
\hline Edad & $129(125$ a 134$)$ & $<0,001$ \\
$\quad<65$ años & $100(91$ a 108$)$ & \\
$\quad \geq 65$ años & $122(117$ a 1127$)$ & $<0,001$ \\
\hline Tipo de cáncer & $66(50$ a 81$)$ & \\
Endometroide & & $<0,001$ \\
No endometroide & $130(124$ a 135$)$ & \\
\hline Grado & $100(89$ a 112$)$ & \\
G1 & $95(83$ a 107$)$ & \\
G2 & & \\
G3 & $124 / 119$ a 130$)$ & \\
\hline Estadio FIGO & $81(67$ a 95$)$ & \\
I & $77(59$ a 95$)$ & \\
II & $49(30$ a 67$)$ & \\
III & & \\
IV &
\end{tabular}

* Media de supervivencia al no poderse estimar la mediana en todos estratos 
3.2 Análisis multivariante (Regresión de Cox)

\begin{tabular}{lcc}
\hline & HR (IC95\%) & p \\
\hline $\begin{array}{l}\text { Abordaje } \\
\text { Laparotomía (ref) } \\
\text { Laparoscopia }\end{array}$ & $0,89(0,49-1,65)$ & 0,72 \\
\hline Edad (incr. 1 año) & $1,047(1,014-1,080)$ & 0,005 \\
\hline Tipo de cáncer & & 0,27 \\
$\quad$ Endometroide (ref) & 1 & \\
$\quad$ No endometroide & $1,53(0,72-3,26)$ & \\
\hline Grado & & 0,001 \\
I (ref) & 1 & \\
II & $8,49(1,95-36,9)$ & \\
III & $17,0(3,74-77,7)$ & \\
\hline Estadio FIGO & 1 & 0,001 \\
I (ref) & $0,74(0,21-2,48)$ & \\
II & $2,38(1,24-4,57)$ & \\
III & $6,36(2,13-19,0)$ & \\
IV & & \\
\hline
\end{tabular}

Una quinta parte de las pacientes fallecieron a lo largo del seguimiento, un porcentaje mayor en el grupo de laparotomía sin ajustarlo por otros factores determinantes. El 11,7\% (52 pacientes) fallecieron por el adenocarcinoma de endometrio mientras un 8,5\% (38 pacientes) fallecieron por otras causas.

Por lo tanto en el análisis multivariante se comprobó que el tiempo libre de enfermedad y la supervivencia no dependen del tipo de abordaje quirúrgico realizado. Una mayor edad, el grado histológico y el estadio de la enfermedad son factores de riesgo que incrementan el riesgo de recaída, acortando el tiempo libre de enfermedad y en definitiva disminuyendo la supervivencia.

\section{DISCUSIÓN}

Nuestros resultados muestran que la laparoscopia es una técnica quirúrgica segura en el tratamiento del adenocarcinoma de endometrio tanto en el tipo endometrioide como en el no endometrioide. Podemos afirmar que este abordaje quirúrgico no afecta a la supervivencia ni al tiempo libre de enfermedad.
Estos hallazgos nos permiten continuar con dicha técnica en el Complejo Hospitalario de Navarra, ya que no se verá afectada la seguridad oncológica en estas pacientes y se seguirán beneficiando de las ventajas que ofrece la vía laparoscópica como es la disminución de la estancia hospitalaria y la precoz vuelta a la vida cotidiana.

Como limitaciones cabe destacar el sesgo temporal, ya que la laparoscopia se fue incorporando tras años de empleo de la laparotomía, por lo que en ocasiones el realizar una técnica u otra no es aleatorio sino que va relacionado con el año en que se realizó el tratamiento. Pero a su vez el tamaño muestral disponible permitirá detectar diferencias como estadísticamente significativas.

En la actualidad se acepta la seguridad de la vía laparoscópica en estadios iniciales de adenocarcinoma de endometrio ${ }^{2-8}$ no quedando tan claro en estadios más avanzados donde el grupo de Palomba y col consideran que se puede conllevar un abordaje subóptimo ${ }^{9}$ no apoyado por otros grupos de trabajo ${ }^{10}$. Todos estos grupos de trabajo son grupos de referencia a nivel mundial ${ }^{11,12}$ por lo que es importante contrastar estos datos con los obtenidos en 
la sección de Ginecología Oncológica del Complejo Hospitalario de Navarra. Nuestros datos globales muestran que no se afecta la supervivencia ni el tiempo libre de enfermedad por el abordaje quirúrgico realizado.

Dado el pronóstico y características de la enfermedad la mayor parte de los casos se tratan de adenocarcinomas de endometrio en estadios precoces por lo que el resto de los casos son un pequeño número lo que dificulta establecer conclusiones. Esto ocurre en la mayor parte de grupos de trabajo $^{2-8,13}$.

Comprobar la seguridad oncológica y el abordaje quirúrgico óptimo de la laparoscopia en estadios avanzados de dicha enfermedad, puede ser el objetivo de próximas investigaciones.

\section{Agradecimientos}

Agradecimientos a la Fundación Miguel Servet así como al grupo de análisis estadístico AIDICS por su apoyo en el análisis estadístico.

Agradecimiento al doctor Gregorio Tiberio por su apoyo en la elaboración.

\section{BIBLIOGRAFÍA}

1. Cáncer de endometrio: Tratamiento. National Cancer Institute. Junio 2012.

2. Galaal K, Bryant A, Fisher AD, Al-Khaduri M, KEW F, LOPES AD. Laparoscopy versus laparotomy for the managementbof early stage endometrial cancer. Cochrane Database of Systematic Reviews 2012, Issue 9. Art. No: CD006655.

3. Palomba S, Falbo A, Mocciaro R, Russo T, Zullo F. Laparoscopic treatment for endometrial cancer: a meta-analysis of randomized controlled trials (RCTs). Gynecol Oncol 2009; 112: 415-421.

4. Acholonu UC JR, Chang-Jackson SC, Radjabi AR, NEZHAT FR. Laparoscopy for the management of early-stage endometrial cancer: from ex- perimental to standard of care. J Minim Invasive Gynecol 2012; 19: 434-442.

5. Taskin S, Güngor M, Öztuna D, Ortaç F. Comparision of laparoscopy and laparotomy in surgical staging of clinical early stage endometrial cancer: a report of early experience from Turkey. J Obstet Gynaecol 2012; 32: 687690.

6. Perrone AM, Di Marcoberardino B, Rossi M, Pozzati F, Pellegrini A, Procaccini M et al. Laparoscopic versus laparotomic approach to endometrial cancer. Eur J Gynaecol Oncol 2012; 33: 376-381.

7. Mourits MJ, Bijen CB, Arts HJ, ter BrugGe HG, VAN DER SIJde R, PAULSEn L et al. Safety of laparoscopy versus laparotomy in early-stage endometrial cancer: a randomized trial. Lancet Oncol 2010; 11: 763-771.

8. Momeni M, Kolev V, Cardenas-Goicoechea J, GetraJdman J, Fishman D, Chuang L et al. Does the type of surgery for early-stage endometrial cáncer affect the rate of reported lymphovascular space invasion in final pathology specimens? Am J Obstet Gynecol 2013; 208: 71.

9. Palomba S, Ghezzi F, Falbo A, Mandato VD, AnNUNZIATA G, Lucia E et al. Laparoscopic versus abdominal approach to endometrial cáncer: a 10-year retrospective multicenter analysis. Int J Gynecol Cancer 2012; 22: 425-433.

10. Lu Z, Yi X, Feng W, Ding J, Xu H, Zhou X et al. Cost-benefit analysis of laparoscopic surgery versus laparotomy for patients with endometrial cancer: Experience from an institute in China. J Obstet Gynaecol Res 2012; 38: 1011-1017.

11. Ball A, Bentley JR, O’Connell C, Kieser Ke. Choosing the right patient: planning for laparotomy or laparoscopy in the patient with endometrial cancer. J Obstet Gynaecol Can 2011; 33: 468-474.

12. CARTER JR. Laparoscopy or laparotomy for endometrial cáncer? A review of three prospective randomized trials. Aust $\mathrm{N} Z \mathrm{~J}$ Obstet Gynaecol 2011; 51: 387-392.

13. Obermair A, Janda M, Baker J, Kondalsamy-ChenNAKESAVAn S, BRAnd A, Hogg $R$ et al. Improved surgical safety after laparoscopic compared to open surgery for apparent early stage endometrial cancer: results from a randomised controlled trial. Eur J Cancer 2012; 48: 11471153. 